$\underline{\text { Conferences }}>\underline{2017 \text { IEEE 4th International C... }}$

\title{
The world-first deployment of narrowband loT for rural hydrological monitoring in UNESCO biosphere environment
}

\section{Publisher: IEEE}

9

Author(s)

Rosdiadee Nordin; Hafizal Mohamad; Mehran Behjati ; Anabi Hilary Kelechi; Nordin

Ramli ; Kentaro Ishizu; Fumihide Kojima; Mahamod Ismail ; Mushrifah Idris

Paper

Citations

228

Full

Text Views

Abstract

Authors

Figures

References

Citations

Keywords

Metrics

More Like This

- Download PDF

- Download Citation

- View References

- Email

- Request Permissions

- Export to Collabratec

- $\quad$ Alerts

Abstract: The success of a rural wireless monitoring system depends on establishing a reliable wireless link over the TCP/IP communication protocol in a challenging terrain and elevation profile. Several studies have shown that link reliability in a rural area can neither be predicted with high accuracy nor precisely modeled using existing mathematical channel modeling tools. Hence, the use of the empirical approach to infer wireless link reliability. This work focuses on the revival of a rural hydrological/water monitoring system with emphasis on the wireless link located in Tasik Chini, a lake with UNESCO biosphere status. The contributions of this study include: understudy the link reliability of a centralized wireless sensor network infrastructure system using the $2 \mathrm{G}$ and Long Range (LoRa) wireless network, the performance limitation of the low data wireless sensor network in a rural environment, approaches to revive rural water station monitoring center and finally highlight potential opportunities in rural wireless communications. View less Metadata 


\section{:三Contents}

\section{Introduction}

Wireless sensor networks (WSNs) are an integral part of any remote monitoring system as it provides an amenable strategy to automate data collection with less human and material cost [1]. The system design of Tasik Chini hydrological monitoring center is based on WSNs infrastructure. Consequently, for Tasik Chini hydrological monitoring center to operate optimally, the reliable wireless link is crucial. As a matter of fact, this was the main problem that leads to near system collapse before this project set out to resuscitate the center.

\section{Sign in to Continue Reading}

\section{More Like This}

A reliable transport protocol for Wireless Sensor Networks

2008 International Symposium on Telecommunications

Published: 2008

PORT: a price-oriented reliable transport protocol for wireless sensor networks

16th IEEE International Symposium on Software Reliability Engineering (ISSRE'05)

Published: 2005

Top Organizations with Patents on Technologies Mentioned in This Article

View More

\section{IEEE Personal Account}

\section{Purchase Details}

A not-for-profit organization, IEEE is the world's largest technical professional organization dedicated to advancing technology for the benefit of humanity.

(C) Copyright 2020 IEEE - All rights reserved. Use of this web site signifies your agreement to the terms and conditions. 